\title{
Dear Reader
}

As we approach the end of 2015, and publish our final issue of Clinical Pharmacokinetics for the year, the editors and production staff at Adis wish to reflect on another successful year's achievements and pay thanks to all those who have contributed their time and effort to guarantee the exceptional quality of our content.

We are delighted to have seen the conclusion of formalities surrounding the joint venture of Springer Science + Business Media with Nature Publishing Group, Palgrave Macmillan, Macmillan Education in early May. Substantial ground has already been made in the integration of business within the resulting Springer Nature company and we at Adis look eagerly toward the future, as the benefits of the collective expertise and capabilities of this company carry us forward.

The majority of our journals saw a rise in ISI impact factor against the previous year. Most notably, BioDrugs increased by $41 \%$ to 2.989; the European Journal of Drug Metabolism and Pharmacokinetics increased $19 \%$ to 1.563; CNS Drugs increased by $17 \%$ to 5.113; Targeted Oncology increased $16 \%$ to 4.000; Pediatric Drugs increased $15 \%$ to 1.975 ; Drugs \& Aging increased $13 \%$ to 2.838; and Molecular Diagnosis \& Therapy increased a further $12 \%$ to 2.891, having already jumped $53 \%$ in the preceding year. We hope to build upon these gains, once more, in 2016.

The Adis Book programme has grown substantially and 20 new titles will have been published over the course of the year. Over 50 further titles are currently in development with leading academics, internationally, and we continue to add more to the list. Our thanks, again, to all who have submitted these valuable proposals to date.

We offer our sincere thanks to all authors who have contributed articles to Clinical Pharmacokinetics over the course of 2015. Their skill and dedication are critical to the continued publication of the journal. The world-class quality of published articles is, similarly, testament to the significant efforts of the peer reviewers, whose commitment ensures that our content is held to the highest possible standard. In addition to the members of our Honorary Editorial Board, we would like to thank the following individuals who acted as reviewers for articles published in Clinical Pharmacokinetics in 2015:

Khaled Abduljalil, UK

Darrell R. Abernethy, USA

Luca Agnifili, Italy

Jane Alcorn, Canada

Jan-Willem C. Alffenaar, The Netherlands

Karel Allegaert, Belgium

Deepak N. Amarapurkar, India

Guohua An, USA

Peter Anderson, USA

Brian J. Anderson, New Zealand

Maria Andersson, Sweden

Pieter Annaert, Belgium

Paul Arnouts, Belgium

Anders Asberg, Norway

Sanjula Baboota, India

David J. Back, UK
Kyun-Seop Bae, Republic of Korea

Judith Bellapart, Australia

Leslie Z. Benet, USA

Eva Benfeldt, Denmark

Sébastien Benzekry, France

Ahmad Beydoun, Lebanon

Agnieszka A. Bienert, Poland

Eliane M. Billaud, France

Scott S. Billecke, USA

Nathalie Bleyzac, France

Alan V. Boddy, Australia

Marta Boffito, UK

Sieto Bosgra, The Netherlands

Marie Bretaudeau Deguigne, France

David C. Broadway, UK

Dion R. Brocks, Canada 
Thierry Buclin, Switzerland

Núria Buil-Bruna, Spain

David M. Burger, The Netherlands

Mieke Carlier, Belgium

Dario Cattaneo, Italy

Etienne Chatelut, France

Vidya Chidambaran, USA

Shripad D. Chitnis, USA

Mette Marie H. Christensen, Denmark

Uwe Christians, USA

Jae-Yong Chung, Republic of Korea

James C. Cloyd, USA

Johan F. Coetzee, South Africa

Angela Colbers, The Netherlands

Sandra D. Comer, USA

Timothy R. Cressey, USA

David Czock, Germany

Youssef Daali, Switzerland

Ann K. Daly, UK

Anthony P. Davenport, UK

Clara T. M. M. de Kanter, The Netherlands

Elizabeth C. M. de Lange, The Netherlands

Jose de Leon, USA

Saskia N. de Wildt, The Netherlands

Brian Scott Decker, USA

Xavier Decleves, France

Paolo Denti, South Africa

Paul P. Dobesh, USA

Matthew P. Doogue, New Zealand

Juergen Drewe, Switzerland

Ana I. Duarte, Portugal

L. Lee Dupuis, Canada

Thomas Ebner, Germany

Andrea Edginton, Canada

Raymond Evers, USA

Agata Faron-Gorecka, Poland

Edward Faught, USA

Liang Feng, USA

Christopher N. Floyd, UK

Kwong M. Fock, Singapore

Qiang Fu, USA

Uwe Fuhr, Germany

Masahide Fukudo, Japan

Takahisa Furuta, Japan

Andrea Gaedigk, USA

Alexsandra Galetin, UK

James M. Gallo, USA

Marta Garaulet, Spain

Maria J. Garcia, Spain

Iain Gardner, UK

Aurelie Gautier, Switzerland

Marc Ghannoum, Canada

Kathleen M. Giacomini, USA
Pascal Girard, Switzerland

Navin Goyal, USA

Garry G. Graham, Australia

Manuela D. Grimstein, USA

Andreas H. Groll, Germany

Martin Grossherr, Germany

Henk-Jan Guchelaar, The Netherlands

Howard Gurney, Australia

Lars Gustafsson, Sweden

Richard I. Hall, Canada

Karen L. Hardinger, USA

John P. Harrelson, USA

Manuel Haschke, Switzerland

Moustapha Hassan, Sweden

Handan He, USA

Alison E. Heald, USA

Georg Hempel, Germany

Stefanie Hennig, Australia

Christoph Hiemke, Germany

Fenglei Huang, USA

Alwin D. R. Huitema, The Netherlands

Steen E. Husted, Denmark

Paul R. Hutson, USA

Ichiro I. Ieiri, Japan

Ibrahim Ince, Germany

Yushi Inoue, Japan

Martin Jönsson, Sweden

Evelyne Jacqz-Aigrain, France

Sarayut Janmahasatian, Thailand

Alexander A. Jetter, Switzerland

Markus Joerger, Switzerland

Susanne Johansson, UK

Trevor N. Johnson, UK

Hannah M. Jones, USA

Siv Jonsson, Sweden

William J. Jusko, USA

Leonid Kagan, USA

Kohei Kaku, Japan

Thomas Kakuda, USA

Sanjay Kalra, India

J. Cory Kalvass, USA

Ron Keizer, Sweden

Saye Khoo, UK

Jennifer J. Kiser, USA

Catherijne A. Knibbe, The Netherlands

Shinji Kobuchi, Japan

Remco Koster, The Netherlands

Stephan Krahenbühl, Switzerland

Elke H. J. Krekels, The Netherlands

Jacek Kubica, Poland

Dirk R. J. Kuypers, Belgium

Cornelia B. Landersdorfer, Australia

Graham Lappin, UK 
Sze, W. J. Lau, USA

Hendrikus J. M. Lemmens, USA

Scott S. L. Letendre, USA

Francis Levi, France

Russel E. Lewis, Italy

Jia Li, China

Jeffrey Lipman, Australia

Xiao-Dong Liu, China

Rocío Lledó-García, Sweden

Elaine Lo, Canada

Yuan Lu, USA

Sreeraj Macha, USA

Rajanikanth Madabushi, USA

Howard I. Maibach, USA

Roberto Mandrioli, Italy

Amelie Marsot, France

Jennifer H. Martin, Australia

Catia Marzolini, Switzerland

Thibaud Mathis, France

Massimo Carlo Mauri, Italy

Jean-Xavier Mazoit, France

Helen McIlleron, South Africa

Andrew J. McLachlan, Australia

Murad R. Melhem, USA

Giovanni Merlino, Italy

Gerd Mikus, Germany

Jose Molto, Spain

Mario Monshouwer, Belgium

David E. Moody, USA

Miriam Mooij, The Netherlands

Samer Moukassi, Canada

Mashio Nakamura, Japan

Rajesh Narwal, USA

Michael A. Nauck, Germany

Elisabet I. Nielsen, Sweden

Dag Nyholm, Sweden

Ryuichi Ogawa, Japan

Hiroshi Omote, Japan

Mehdi Oualha, France

Stuart W. Paine, UK

Dominic L. Palazzolo, USA

Willia M. Pardridge, USA

Neil J. Parrott, Switzerland

Jignesh P. Patel, UK

Bhaumik, B. Patel, USA

Ewan Pearson, UK

Vidya Perera, USA

Juan José Perez-Ruixo, Spain

Leonor Periáñez-Párraga, Spain

Marc Pfister, Switzerland

Elodie L. Plan, Sweden

J. Robert Powell, USA

Aurelie Premaud, France
Eyas Raddad, USA

Isabelle D. Ragueneau-Majlessi, USA

Muhammad F. Rasool, Germany

Michael D. Reed, USA

David M. Reith, New Zealand

Michael J. Rieder, Canada

Christoph A. Ritter, Germany

Jason A. Roberts, Australia

Keith A. Rodvold, USA

Felix Rosenow, Germany

Colin Ross, Canada

Amin Rostami-Hodjegan, UK

Malcolm M. Rowland, UK

Andrew Rowland, Australia

Hassane Sadou Yaye, France

Sanjeeb S. K Sahoo, India

Smita Salunke, UK

André J. Scheen, Belgium

Gaia Schiavon, UK

Matthias Schwab, Germany

Sorell Schwartz, USA

Catherine M. T. Sherwin, USA

Marco Siccardi, UK

Katrin Sikk, Estonia

Ulrika Simonsson, Sweden

Jan Snoeys, Belgium

Edoardo Spina, Italy

Georgina Sposetti, Argentina

Christine E. Staatz, Australia

Christophe Stove, Belgium

Michael A. Ströhlein, Germany

Bruno H. Stricker, The Netherlands

Mitsushige Sugimoto, Japan

Yan V. Sun, USA

Ulrika S. H. Svensson, Sweden

Anne-Marie Taburet, France

Prue Talbot, USA

Nikhil Tandon, India

Kunal Taskar, UK

Jacquelyn Taylor, USA

Robert ter Heine, The Netherlands

David Ternant, France

Huybrecht T'Jollyn, Belgium

Ivan Tkac, Slovakia

Roger D. Toothaker, USA

Aleksi Tornio, Finland

Vijay V. Upreti, USA

Muthiah Vaduganathan, USA

Volker Vallon, USA

Teun Van Gelder, The Netherlands

Manthena V. Varma, USA

Karthik Venkatakrishnan, UK

Svante Vikingsson, Sweden 
Jean-Louis Vincent, Belgium

Georgios Vlasakakis, UK

Winnie Vogt, Germany

Suzanne Vosburg, USA

Junwen Wang, China

Nigel J. Waters, USA

Dietmar Weitz, Germany

Andrés J. Yarur, USA

Graeme Young, UK
Malcolm A. Young, USA

Maciej J. Zamek-Gliszczynski, USA

Jianping Zhang, USA

Lillian Zhang, USA

Zvetanka Zhivkova, Bulgaria

Michael A. Zientek, USA

Mingxin Zuo, USA

Athena F. Zuppa, USA

We hope that you have found the articles published throughout 2015 to be both interesting and informative. The editors have appreciated the high quality of content contributed to the journal this year and look forward to keeping you up to date with topical issues in the field of clinical pharmacokinetics in 2016.

With best wishes from the staff of Clinical Pharmacokinetics and all at Adis Publications. 